\title{
Montaigne: un regard mathématique sur la mort
}

MARCEL GOULET

À la mémoire de mon père.

Résumé: La réflexion que, dans les Essais, Montaigne poursuit sur la mort, en vue de découvrir unars moriendi spécifiquement humain - toute croyance religieuse étant expressément écartée -, l'amène à soumettre à son jugement la doctrine stoïcienne et sa rhétorique de l'amenuisement, d'une part, et l'attitude commune et sa rhétorique de l'ignorance, d'autre part. L'exercice reconduira Montaigne à l'irrésolution, non sans qu'il ait mis en évidence, chemin faisant, les figures mathématiques que l'on associe à la mort, de la division à la soustraction, de l'addition à la multiplication.

\section{$\mathrm{L}$}

a méditation que Montaigne pratique au sujet de la mort provient, dans les Essais, ${ }^{1}$ d'une préocupation d'ordre éthique. Montaigne se demande comment "bien mourir" et, pour ce faire, quelle attitude adopter à l'égard de la mort. Il espère pour lui-même une mort douce, "la plus legere et aucunement delicieuse" (III:13,1081c) qui soit, une mort "heureuse" (I:20,95a); et il est en quête d'un ars moriendi qui réponde à cette exigence. Il est cependant décidé à ne conduire sa recherche que dans une perspective résolument humaine. ${ }^{2} \mathrm{Le}$ fait n'est pas singulier: tous les Essais procèdent de cette volonté. Sa résolution le conduira ici à la mise en veilleuse, non pas nécessairement au rejet, du discours religieux, à la mise à l' "essai" de la doctrine stö̈cienne, à l'examen, enfin, de l'attitude "vulgaire" ou "naturelle" à l'égard de la mort.

Pourquoi, de prime abord, Montaigne procède-t-it à la mise à l'écart du discours religieux et de l'autorité divine qui le fonde? Ce discours présente 
certes un attrait particulier pour qui s'interroge sur le sens de la mort dans le but de trouver la meilleure conduite à adopter à l'égard de l'événement. Le discours catholique, celui que connaît Montaigne, propose un ars moriendi fondé sur les idées de Providence et d'immortalité. Il promet la béatitude éternelle à celui qui mène une existence vertueuse, sait reconnaître ses fautes et à travers la pénitence, arrive à purifier son âme. Le discours est rassurant, d'autant que le Dieu qui en est le garant est un Être de miséricorde. Peut-on espérer art de mourir plus réconfortant que celui où la mort apparaît comme le passage vers une autre vie, heureuse et éternelle? Pourquoi Montaigne refuset-il à son esprit inquiet ce refuge que lui procurerait l'adhésion au récit chrétien du salut? C'est que l'adoption, d'entrée de jeu, d'une telle réponse à son interrogation entre en contradiction avec le projet même des Essais: cela dispenserait Montaigne de l'exercice spécifiquement humain de son jugement, exercice à travers lequel il rêve de devenir un auctor, au sens fort du terme, c'est-à-dire une autorité, mieux encore sa propre autorité, avec tout ce que l'entreprise comporte de difficulté, voire de "ratés."

La pensée, ainsi mise à l'épreuve sur le mode autonome, quasi autarcique, souhaité par Montaigne, si elle aboutit parfois à la réussite, est le plus souvent reconduite à la reconnaissance de ses limites, du propre aveu de Montaigne:

Le jugement est un util à tous subjects, et se mesle par tout. A cette cause, aux essais que j'en fay ici, j'y employe toute sorte d'occasion. Si c'est un subject que je n'entende point, à cela mesme je l'essaye, sondant le gué de bien loing; et puis, le trouvant trop profond pour ma taille, je me tiens à rive; et cette reconnoissance de ne pouvoir passer outre, c'est un traict de son effect, voire de ceux dequoy il se vante le plus. Tantost, à un subject vain et de neant, j'essaye voir s'il trouvera dequoy lui donner corps et dequoy l'appuyer et l'estançonner. Tantost je le promene à un subject noble et tracassé, auquel il n'a rien à trouver de soy, le chemin en estant si frayé qu'il ne peut marcher que sur la piste d'autruy. Là il fait son jeu à eslire la route qui luy semble la meilleure, et, de mille sentiers, il dict que cettuy-cy, ou celuy là, esté le mieux choisi.

(I:50,289a/c)

D' aucuns, cependant, surtout en un certain dix-septième siècle fort catholique, jugeront l'attitude présomptueuse, orgueilleuse et, du coup, scandaleuse. Je ne vois pour ma part, dans cette suspension du discours religieux sur le sujet de la mort, qu'une sorte de réduction phénoménologique à la Husserl, ${ }^{3}$ une mise en veilleuse qu'il ne faudrait surtout pas confondre avec un déni, puisqu'elle est fondée, chez celui qui l'opère, sur la reconnaissance d'un statut d'autorité privilégié à la Parole de Dieu: 
Si philosopher c'est douter, comme ils disent, à plus forte raison niaiser et fantastiquer, comme je fais, doit estre doubter. Car c'est aux apprentifs à enquerir et à debatre, et au cathedrant de resoudre. Mon cathedrant, c'est l'authorité de la volonté divine, qui nous reigle sans contredit et qui a son rang au dessus de ces humaines et vaines contestations.

Pour Montaigne, om le voit bien, la parole humaine ne saurait être mise en compétition avec la Révélation divine. La Parole divine est de l'ordre de la résolution: elle s'exprime par arrêts, au sens juridique du terme. L'esprit humain, au contraire, se meut dans l'irrésolution; à ce titre, il est condamné à ne penser et à ne s'exprimer que par essais. Montaigne sait, de toute manière, qu'au bout de sa démarche il restera toujours pour sa pensée trop humaine, et éventuellement désemparée, le recours possible au Verbe divin. Il a choisi de faire humain. Or, rien de ce qui est humain, pas même la pensée d'Aristote, ne mérite d'être soustrait à l'épreuve du jugement. Restent donc "la doctrine," selon le mot que Montaigne emploie pour désigner la philosophie, et la sagesse populaire. Au cours de l'exercice, pendant lequel ces deux discours feront l'objet d'un examen par son jugement, Montaigne sera amené à considérer un certain nombre d'énoncés à caractère mathématique sur la mort. Je n'entends pas juger de la "réussite" de ses "coup[s] d'essay" (III:9,941b) sur la mort, ni selon les critères que lui-même énonce (I:50,289a/c), ni en termes d'originalité ou de dette, ni en termes de conformité à une orthodoxie philosophique ou religieuse, ou à une quelconque sagesse populaire. Je veux plutôt rendre compte de la présence, dans son discours, de ces propositions mathématiques, et en évaluer la fonction eu égard à la philosophie, à l'attitude commune et au souci de Montaigne d'atteindre à une pensée autonome.

\section{L'“essai” de la philosophie: l’idée de la mort multiple}

L'essai par Montaigne de la philosophie le mènera, tout d'abord, droit à l'examen de la doctrine stoïcienne. De fait, le discours philosophique pris en considération par Montaigne sera pour l'essentiel stoïcien, le stoïcisme d'un Sénèque en particulier, mais accompagné à l'occasion d'idées épicuriennes, par les bons soins d'un Lucrèce que Montaigne aime également fréquenter. Qu' offre donc la doctrine à qui s'interroge sur la mort? Elle propose un ars moriendi fondé sur la dissipation de la crainte de la mort, par la modification de la chose sur laquelle un être humain puisse prétendre jouir d'un certain pouvoir, à savoir son jugement. Le problème de la mort n'est pas tant la mort 
elle-même que la crainte qu'elle inspire, soutient la philosophie. C'est la conscience inquiète de la mort qui est, chez l'homme, la principale source de son tourment, affirment Sénèque et tout autant Lucrèce dans la foulée d'Épicure. Pour bien vivre et se préparer à bien mourir, il faut gagner le repos, la tranquilité, l'état d'ataraxie. La solution se trouve dans l'adoption d'une attitude d'indifférence, d'impassibilité, de détachement à l'égard de la vie. La maîtrise de la sensation, ici de la peur de la mort, passe par le contrôle du jugement. L'opinion apparaît, dans le discours philosophique en cause, comme un champ que se disputent la raison et la sensibilité.

Tout le problème de la mort, aux yeux des philosophes convoqués au débat par Montaigne, vient de l'abdication de la raison, de sa démission face à la sensibilité. La raison, dans la mesure où elle sait se faire dominante, est, comme le dit la doctrine, la clef da la sagesse. Pour assurer l'hégémonie de la raison sur l'opinion, la doctrine philosophique, la stoïcienne en particulier, construira son discours sur une rhétorique — d'aucuns diront sur une sophistique fondée sur des opérations mathématiques, en faisant appel au besoin à la logique et à la physique épicuriennes, et en cherchant à faire la démonstration de l'illégitimité de la crainte de la mort. Le jeu, nous le verrons, n'échappera pas à Montaigne, d'autant qu'il aura lui-même recours à cette rhétorique, et à ses assises mathématiques, pour interpréter sa propre expérience des rapports entre la vie et la mort.

L'homme, selon la philosophie, n'est donc pas tant préoccupé par la mort que par l'opinion qu'il s'en fait, toute mêlée de crainte. Le sage, disent les philosophes, doit arriver à dissiper sa crainte de la mort; il doit, pour ce faire, apprendre à cultiver la nonchalance, le mépris, l'indifférence. Paradoxalement, les philosophes, les Stoïciens surtout, conseillent l'adoption d'une stratégie axée sur la prévoyance, l'apprivoisement, l'attention. La mort doit devenir un sujet de constante préméditation: "n'ayons rien si souvent en la teste que la mort" (I:20, 85a), semble prêcher Montaigne en leur nom; qu'elle devienne, ainsi que chez les philosophes, comme le note Cicéron, " "le but de la vie," "son objet” (III:12, 1028c). Il faut s'accoutumer à elle, soutient encore Montaigne: “Ostons luy [à cet ennemi] l' estrangeté, pratiquons le, accoustumons le" (I:20, $85 \mathrm{a})$; l'accoutumance mène à la connaissance, et la connaissance permet de conjurer la peur. Pour les Stoïciens, en effet, ce n'est pas tant l'ignorance comme la méconnaissance de la chose qui produit l'effarement. L'homme, dont l'opinion est livrée à la sensibilité, est privé de la véritable intelligence de l'objet de ses préoccupations. Aussi ne peut-il faire autrement que de craindre la mort. Seule l'attention, et une attention soutenue, peut le rendre à la raison 
et le mener à l'indifférence à l'égard de la mort. Il faut donc assimiler la mort à la vie: "attendons le partout" (I:20,85a); son affrontement quotidien modifiera, ainsi que César le donne à penser, la perception monstrueuse que nous en avons. "Le mal qu' on a longtemps attendu est plus léger quand il finit par nous toucher," affirme Sénèque. ${ }^{6}$ Le propre de la rhétorique stoïcienne, telle qu'elle se déploie ici, c'est précisément, à l'inverse de cette définition de l'art à laquelle Montaigne se réfère par ailleurs, d'une chose grande la faire paraître petite. ${ }^{7}$ Le fond de la rhétorique stoïcienne, Montaigne commence à le pressentir, est mathématique.

Derrière les prescriptions et les arguments des Stoïciens, Montaigne découvrira de fait une première figure mathématique de la mort. La mort, telle que la représente la doctrine stoïcienne, n'est pas chose une, mais multiple. Elle est une quantité, qui se divise et se fractionne et distribue sa présence, en "declinaisons ordinaires" (I:20,89b), dans les moindres parcelles de la vie. On ne la rencontre jamais entière, elle ne se donne, et fort heureusement, que par fragments:

Qui y tomberoit tout à un coup, je ne crois pas que nous fussions capables de porter un tel changement. Mais, conduicts par sa main, d'une douce pente et comme insensible, peu à peu, de degré en degré, elle nous roule dans ce miserable estat, et nous y apprivoise; si que nous ne sentons aucune secousse, quand la jeunesse meurt en nous qui est en essence et en verité une mort plus dure que n'est la mort entiere d'une vie languissante, et que n'est la mort de la vieillesse.

On croirait bien entendre Sénèque qui, puisant dans le souvenir de ses échanges avec Lucilius, lui écrit:

Je me rapelle que tu as une fois développé l'idée que nous ne tombons pas soudainement dans la mort mais que nous avançons vers elle pas à pas. Nous mourons chaque jour, car chaque jour nous est ôtée une part de notre vie: à mesure que notre âge s'accroît, notre vie diminue. Nous perdons l'enfance, puis l'adolescence, puis la jeunesse: jusqu'à la journée d'hier, tout le temps qui s'est écoulé est mort. ${ }^{8}$

Pourquoi craindrions-nous "une espece de mort, là où nous en avons desjà passé et en passons tant d'autres?" (II:12,587a), se demande un Montaigne apparemment converti à la doctrine stoïcienne. La mort est une série de soustractions qui s'opèrent quotidiennement, imperceptiblement, sur le compte de la vie, jusqu'à un dernier prélèvement, tout aussi minime: 
Pourquoy crains-tu ton dernier jour? Il ne confere non plus à ta mort que chascun des autres. Le dernier pas ne fait pas la lassitude; il la declare. Tous les jours vont à la mort, le dernier y arrive.

Ce n'est que lorsqu'elle laisse échapper la dernière goutte que l'horloge est déclarée vide et que le temps s'arrête. Pourtant, dès le départ de la première goutte, la réserve avait irrémédiablement commencé à s'épuiser.

Ce n'est pas la dernière goutte qui vide la clepsydre, rappelle Sénèque à Lucilius, mais toutes celles qui sont tombées auparavant: ainsi la dernière heure, celle de notre fin, n'est pas la seule à provoquer notre mort, mais la seule à la mener à terme. C'est à ce moment que nous atteignons le but, mais nous marchons depuis longtemps. ${ }^{9}$

La vie est un crédit dont la mort est le débit: "Tout ce que vous vivez, vous le desrobez à la vie; c'est à ses despens" (I:20,91c). La vie et la mort sont des compagnes quotidiennes, familières, à toutes fins utiles, permutables: "Vous estes en la mort pendant que vous estes en vie" (I:20,91c). Selon la logique stoïcienne, en effet, la mort entretient avec la vie un rapport d'inclusion, voire d'équivalence: "c'est une partie de vous que la mort [. . . Cettuy vostre estre, que vous joüyssez, est egalement party à la mort et à la vie" (I:20,91a). "La mort se mesle et confond par tout à nostre vie: le declin praeoccupe son heure et s'ingere au cours de nostre avancement mesme" (II:13, 1082b). "Même le jour que nous sommes en train de vivre, écrivait jadis Sénèque, nous le partageons avec la mort!"10 La vie n'est en fin de compte, pour les Stoïciens, qu'une suite de morts partielles, infinitésimales. "Il n'y a pas qu'une mort, rappelait Sénèque à Lucilius en reprenant ses propres paroles; mais celle qui nous emporte est la mort ultime." "La rhétorique stoïcienne ne paraît emprunter à la mathématique que pour davantage rapetisser la mort, la réduire, l'amenuiser, que pour mieux conjurer la crainte qu'on en peut avoir. Montaigne découvre là, à la lumière de la pensée stö̈cienne et quelque trois siècles avant Bouvard et Pécuchet, ${ }^{12}$ la vertu dissicative de la mathématique. La doctrine stoïcienne de la mors cotidiana et de la mors vitalis enlève pratiquement tout son poids à l'ultime mort. Quelle sensibilité daignerait s'émouvoir devant une opération devenue dès lors si infime, si banale? Le coeur étiolé, l'adversire mis à sec, la raison désormais occupe seule l'opinion et peut à loisir la gagner à sa cause.

La figure tout arithmétique de la mort, trouvée par Montaigne dans les replis de la pensée stoïcienne, lui servira à interpréter sa propre expérience de la vie: 
Voilà une dent qui me vient de choir, sans douleur, sans effort: c'estoit le terme naturel de sa durée. Et cette partie de mon estre et plusieurs autres sont desjà mortes, autres demy mortes, des plus actives et qui tenoient le premier rang pendant la vigueur de mon aage. C'est ainsi que je fons et eschape à moy

(III:13,1081b)

Montaigne éprouve ici tout le profit de la considération de la mort comme opération de soustraction. Il vient un temps où, tout compte fait, sa vie ayant été nombre de fois débitée, l'être humain ne s'appartient plus, puisque dès lors il appartient davantage à la mort qu'à la vie. Il y a un moment où, s'aperçoit Montaigne à la lumière de la rhétorique stoïcienne, l'être bascule dans le nonêtre. "Ce que je seray doresenavant, admettra-t-il un jour avec un brin de nostalgie, ce ne sera plus qu'un demy estre, ce ne sera plus moy. Je m'eschape tous les jours et me desrobe à moy" (II:17,625a). Il se laissera tout de même quelque peu séduire par cette figure soustractive de la mort, le temps à tout le moins d'en espérer une dernière mort d'autant plus légère: "Dieu faict grace à ceux à qui il soustrait la vie par le menu; c'est le seul benefice de la vieillesse. La dernière mort en sera d'autant moins plaine et nuisible; elle ne tuera plus qu'un demy ou un quart d'homme" (III:13,1081b). La mort, en effet, paraît d'autant plus grande et effrayante, injustifiable même, qu'elle frappe en l'enfance ou en la jeunesse. Elle emporte alors son plein lot de vie. Mais, plus on vieillit, moins on meurt, sommes-nous tentés de conclure!

Si la division de la mort a pour bénéfice son ultime banalisation, il n'en reste pas moins que cet allégement de la dernière mort par la répartition de son poids sur la durée de la vie a un effet pervers, et Montaigne le sait: l'apprêt à la mort s'en trouve, lui, alourdi d'autant. Montaigne estimera donc, en dernière instance, que la philosophie stö̈cienne ne fait que substituer, dans la balance de l'opinion, un poids à un autre: au poids de la dernière mort et de la crainte qu'elle suscite, elle substitue, voire surajoute - Montaigne en prend à témoin Aristote, "lequel la mort presse doublement, et par elle, et par une si longue prevoyance" (III:12,1029b) —, le poids de la préparation mentale et des morts multiples. Car “[i]l est certain qu'à la plus part, la preparation à la mort a donné plus de tourment que n'a faict la souffrance” (III:12,1028b). Montaigne préférera se rendre à l'opinion de César, pour lequel "la moins pourpensée mort estoit la plus heureuse et plus deschargée” (III:12,1029b). Et puis l'idée de la mors cotidiana et de la mors vitalis ne lui paraît pas, tout compte fait, chose si désirable: "Je voyois nonchalamment la mort, quand je la voyois universellement, comme fin de la vie; je la gourmande en bloc; par le menu, elle me pille" (III:4,815c). La mort divisée, mais partant plus assidue, paraît, 
au rebours de l'effet recherché, n'avoir fait que décupler son pouvoir d'exaspération. La rhétorique stoïcienne, en recourant à la mathématique, semble finalement s'être égarée, aux yeux de Montaigne, dans de bien mauvais calculs. La fréquentation quotidienne de la mort, qui était censée en procurer une connaissance rédemptrice de la crainte qu' elle inspire, et sa répartition par division sur la multitude des jours n'auront pas su remplir leur promesse, paradoxale, de nonchalance.

\section{L'“essai” de l'attitude commune: l'idée de la mort unique}

Montaigne examine également, presque simultanément, l' attitude vulgaire à l'égard de la mort. L'homme du commun, constate-t-il, n'a pas besoin de cultiver la nonchalance; il la pratique d'instinct, naturellement:

Je ne vy jamais paysan de mes voisins entrer en cogitation de quelle contenance et asseurance il passeroit cette heure derniere. Nature luy apprend à ne songer à la mort que quand il se meurt.

Montaigne découvre qu'une telle conduite ne peut s'expliquer que par une rhétorique de l'insouciance, de la fuite, de l'oubli. La mort et son apprêt, mental et matériel, sont pour ces êtres du commun, tels un "valet" ou une "chambriere," les moindres de leurs soucis: "Le remede du vulgaire, c'est de n'y penser pas" (I:20,82a). Il suffit d'en détourner son attention: la diversion mène à l'ignorance et ce qu' on ignore ne fait pas de mal. "A quoy faire la cognoissance des choses, si nous en perdons le repos et la tranquilité, où nous serions sans cela, et si elle nous rend de pire condition que le pourceau de Pyrrho" (I:14,54-55a)? Ne serait-il pas préférable de conduire sa vie en l'absence de toute préoccupation à l'égard de la mort?

Derrière cette attitude d'une simplicité toute naturelle, et sa rhétorique de l'ignorance, Montaigne décèle une autre figure mathématique de la mort. La mort est, pour le vulgaire, chose une et infrangible; elle n'arrive qu'une fois et entière, et: "Rien ne peut estre grief, qui n'est qu'une fois" (I:20,90c), dira un Montaigne devenu, semble-t-il, partisan du commun. Elle appartient à l'ordre de la brièveté, de l'instant, un instant où elle se concentre toute: “c'est chose trop momentanée. Un quart d'heure de passion sans consequence, dans nuisance, ne merite pas des preceptes particuliers”(III:12,1028b/c). “Cen'estqu'un instant, objecte Montaigne; mais il est de tel pois" (III:9,962b). Pourtant, se demande-t-il, prêtant à nouveau sa voix au vulgaire: "Est-ce raison de craindre si long temps chose de si brief temps?" (I:20,90c). Et puis, selon la logique commune, le rapport de la mort à 
la vie en est un d'exclusion: elle n'en est que "le bout," l'“estremité" (III:12,1028c). Et elle se trouve bien là: laissons-y!

La rhétorique de l'ignorance, propre au vulgaire, n'a certes pas besoin de justification: elle se pratique d'instinct. Cela n'empêche pas Montaigne, cependant, de s'interroger sur la légitimité de cet ars moriendi. L'examen de l'attitude commune, de la perception de la mort comme événement unique, situé à la limite de la vie, dans un rapport de quasi exclusion avec elle, conduira Montaigne à renouer avec la doctrine et à constater qu'elle est porteuse d'autres figures mathématiques de la mort, figures présentes dans le discours philosophique d'un Épicure ou d'un Lucrèce, mais qui s'accordent mieux avec l'ars moriendi populaire que les idées de mors vitalis et de mors cotidiana, fondées sur la relation d'inclusion de la mort dans la vie, et chères à la doctrine stoïcienne. L'attitude commune, qui voit dans la mort un fait unique, bref et instantané, et qui ne lui connaît d'autre rapport avec la vie que celui d'en être la fin, s'accommode fort bien, en effet, de l'idée de la mort comme passage, un passage à une autre vie, ou comme renaissance, une naissance à un nouveau mode d'être au monde. Il y a, pour l'homme, croit Épicure, deux façons d'être au monde: le mode de la présence, acquis à la naissance, et cet autre mode, plutôt paradoxal, de l'absence, que procure la mort. À la vie présente, au cours de laquelle l'homme jouit de la sensation des choses, succéderait une autre vie où il serait privé de cette sensation.

Comme nostre naissance nous apporta la naissance de toutes choses, observe Montaigne devenu cette fois épicurien pour le besoin de la chose, aussi fera la mort de toutes choses nostre mort. Parquoy c'est pareille folie de pleurer de ce que d'icy à cent ans nous ne vivrons pas, que de pleurer de ce que nous ne vivions pas il y a cent ans. La mort est origine d'une autre vie.

(I:20,90c)

La mort est bel et bien ici conçue comme un ajout à la vie, comme l'événement par lequel s'additionne à une première façon, consciente, d'être au monde une manière seconde qui, elle, s'exerce sur le mode de l'inconscience. L'idée que l'homme n'a pas qu'une vie à vivre mais bien deux, la mort se présentant paradoxalement comme une seconde vie où la privation de la sensation rend caducs toute nostalgie et tout souci de la première, justifie fort bien aux yeux de Montaigne que, d'une manière corollaire, on pratique dans l'état premier de la vie une attitude d'insouciance à l'égard de son état second, la mort.

Tout en continuant à s'interroger, avec l'homme du commun et avec Socrate, sur le bien-fondé de la crainte que la mort inspire et sur la légitimité de la rhétorique de l'oubli, Montaigne lui découvrira, grâce à la main secourable 
de Lucrèce, une autre fonction arithmétique:

A quoy faire nous en aurait nature engendré la hayne et l'horreur, veu qu'elle luy tient rang de très-grande utilité pour nourrir la succession et vicissitude de ses ouvrages, et qu'en cette republique universelle elle sert plus de naissance et d'augmentation que de perte ou ruyne? [. . . La defaillance d'une vie est le passage à mille autres vies.

(III:12,1032b)

La mort apparaît ici, sous un autre "lustre," comme une multiplication de la vie. La mort individuelle, bien qu'elle demeure un "acte à un seul personnage" (III:9,957b), est également un événement universel: "Vostre mort est une des pieces de l'ordre de l'univers; c'est une piece de la vie du monde" (I:20,91a). La figure de la mort multiplicatrice repose sur la croyance en l'éternité de la matière et sur l'idée que la mort est un don, un don de matière, par lequel tout individu contribue à la transformation de l'univers, à son mouvement, à sa dynamique, à la régénération de la Nature. C'est ici, à l'arrière-plan, toute la physique de Lucrèce et d'Épicure qui vient se profiler, et dont le principe de départ est que "rien ne peut être engendré de rien."13 Et d'ajouter Lucrèce:

Puisque rien ne peut être créé de rien, je l'ai démontré, et que ce qui est né, le néant ne peut le reprendre, une substance immortelle est donc nécessaire aux éléments en lesquels chaque corps ira se fondre à son heure suprême, afin que la matière puisse suffire au renouvellement incessant du monde. Les corps premiers sont donc simples et solides à la fois; autrement ils ne pourraient résister au temps pour travailler éternellement à la renaissance des êtres. ${ }^{14}$

Ainsi, "la nature réduit chaque corps en ses parties élémentaires, mais ne le fait point périr, ne l'anéantit point." ${ }^{15}$ La mort n'est que la participation matérielle d'un individu à la vie de mille autres individus. En détruisant un être, la nature, soutient Lucrèce, ne fait que réduire cet être en des particules infimes et se sert des débris ainsi produits pour former des êtres nouveaux. ${ }^{16} \mathrm{Du}$ point de vue de l'univers, soustraire équivaut bien à multiplier. Il semble même "que l'université des choses souffre aucunement de nostre aneantissemsnt, ny qu'elle soit compassionnée à nostre estat" (II:13,589a). Serait-ce toujours tentant de comprendre cette crainte que la mort inspire aux uns, cette insouciance où elle en laisse d'autres, "que nous faisons trop de cas de nous" (II:13,589a)? À quoi il répondra: "Nul de nous ne pense assez n'estre qu'un" (II:13,590c), rejoignant par là la logique de l'unité sur laquelle se fonde l'attitude commune à l'égard de la mort: la mort reparaît sous sa figure unique et infrangible, mais transformatrice de l'un en multiple. 
Montaigne est bien tenté d'adhérer à cette vision des choses: “A les juger par l'utilité et la verité naifve les leçons de la simplicité ne cedent gueres à celles que nous presche la doctrine, au contraire" (III:12,1029b). D'autant qu'elle compte, parmi ses "bons regens," Socrate lui-même (III:12,1029b/ 1031b). Qui plus est, elle rencontre son désir: "que la mort me treuve plantant mes chous, mais nonchalant d'elle, et encore plus de mon jardin imparfait" (I:20,87a). Auparavant, toutefois, il s'interroge sur la possibilité d'une telle attitude: "comment est-il possible qu' on se puisse deffaire du pensement de la mort [ . . ]?"'(I:20,84a), allant jusqu'à la croire impossible à tenir à "un homme d'entendement" (I:20,84a). La rhétorique populaire lui paraît, néanmoins, avoir mieux fait ses comptes que la rhétorique stoïcienne.

Les "coups d'essai" de Montaigne sur la mort, inaugurés sous le signe de l'éthique, l'auront conduit, par les chemins de la rhétorique, à la découverte des figures mathématiques de la mort. Relève-t-elle de la division ("tous les jours vont à la mort," I:20,94c), d'une opération par laquelle la rencontre avec la mort est banalisée par sa transformation en une multitude de rendez-vous quotidiens? Est-elle de l'ordre de la soustraction ("privation de la vie," I:20,85c), d'une opération par laquelle la vie est prélevée d'une manière régulière, presque imperceptible, et qui conduit ultimement à la mort d'un nonêtré? Tient-elle de l'addition ("origine d'une autre vie," I:20,90c), d'une opération de passage à un second mode d'être au monde dont tout le bénéfice réside en ce que nul regret du premier n'y soit possible? Ou appartiendrait-elle, enfin, à l'ordre de la multiplication ("le passage à mille autres vies," III:12,1032b), relevant d'une opération créatrice par laquelle la Nature perpétuerait la métamorphose à partir d'une matière éternelle? L'interrogation restera pour Montaigne, par delà les opinions des moralistes et des rhétoriciens, sans réponse arrêtée, emportée par le "que sçay-je?," dans une sorte de résignation à l'irrésolution. Rien ne laisse supposer, en effet, que Montaigne ait cru, fût-ce un instant, avoir contemplé dans ces figures mathématiques l'essence même de la mort. ${ }^{17}$ Il me semble plutôt que, s'il a consenti à reconnaître l'utilité rhétorique et, partant, éthique des figures mathématiques de la mort, il a préféré, quant à leur valeur, s'en tenir au silence.

La métaphore mathématique de la mort n'aura finalement servi qu'à ramener Montaigne de l'éthique vers la métaphysique, à cette ultime question sur la figure que la mort dissimule derrière les masques dont nous la revêtons, à "la consideration de la chose en soy" (III:4,812b), au seuil donc de l'ignorance et de l'incertitude. 


\section{Notes}

1. Non seulement en I:20, mais aussi, entre autres, en I:19, II:3, III:12 et III:13. Toute référence aux Essais est faite d'après l'édition des Oeuvres complètes de Montaigne, texte établi par Albert Thibaudet et Maurice Rat, introduction et notes par Maurice Rat (Paris, Gallimard, "Bibliothèque de la Pléiade," 1962) et mentionne entre parenthèses, dans le corps du texte et dans l'ordre, le livre, l'essai, la page et la couche du texte.

2. Ainsi que l'a bien observé Jules Brody, à qui je suis redevable, par suite de la lecture de son très beau texte, "Montaigne et la mort: deux études sur Que philosopher c'est apprendre à mourir (I,20)" (dans Lectures de Montaigne [Lexington, French Forum, 1982], p. 93-144), de l'idée de ce texte.

3. Sur le recours à l'epochè chez Montaigne, voir André Tournon, Montaigne. La glose et l'essai (Lyon, Presses Universitaires de Lyon, 1983), p. 273, et Montaigne en toutes lettres (Paris, Bordas, 1989), pp. 79 et 81.

4. "Tota philosophorum vita commentatio mortis est" (cité par Montaigne, III:12,1028c).

5. Duquel Montaigne dit tenir l'idée que "les choses nous paroissent souvent plus grandes de loing que de près" (I:20,89a).

6. Sénèque, Lettres à Lucilius. Sur l'amitié, la mort et les livres, préface, traduction et commentaires de Pierre Miscevic (Paris, Presses Pocket, “Agora,” 1990), p. 116.

7. "Un Rhethoricien du temps passé disoit que son mestier estoit, de choses petites les faire paroistre et trouver grandes" (I:51,292a). Quelque direction qu'elle prenne, la rhétorique, ainsi réquisitionnée, demeure pour Montaigne "un" art piperesse et mensongere" (I:51,292a).

8. Sénèque, Op. cit., p. 82.

9. Sénèque, Op. cit., pp. 82-83.

10. Ibid., p. 82.

11. Ibid., p. 83

12. Au mot "mathématiques," dans le "Dictionnaire des idées reçues," Flaubert note pour toute définition: "Dessèchent le coeur" (Bouvard et Pécuchet [Paris, Éditions Gallimard et Librairie Générale Française, "Le livre de poche," 1959/1970], p. 433).

13. Lucrèce, De la Nature, traduction nouvelle, introduction et notes de Henri Clouard, édition revue et corrigée (Paris, Garnier Frères, “Classiques Garnier,”), 1954, p. 11.

14. Ibid., p. 33.

15. Ibid., p. 15.

16. Ibid., pp. 38-39.

17. Cela n'aurait pourtant pas été la seule fois où il se fût découvert une allégeance platonicienne (voir: III:12,1020c). 